\title{
Fidelity decay for local perturbations: Microwave evidence for oscillating decay exponents
}

\author{
Bernd Köber, ${ }^{1}$ Ulrich Kuhl, ${ }^{1,2}$ Hans-Jürgen Stöckmann, ${ }^{1}$ Arseni Goussev, ${ }^{3}$ and Klaus Richter ${ }^{4}$ \\ ${ }^{1}$ Fachbereich Physik der Philipps-Universität Marburg, D-35032 Marburg, Germany \\ ${ }^{2}$ Laboratoire de Physique de la Matière Condensée, CNRS UMR 6622, Université de Nice Sophia-Antipolis, F-06108 Nice, France \\ ${ }^{3}$ School of Mathematics, University of Bristol, University Walk, Bristol BS8 1TW, United Kingdom \\ ${ }^{4}$ Institut für Theoretische Physik, Universität Regensburg, D-93040 Regensburg, Germany
}

(Received 16 September 2010; published 31 January 2011)

\begin{abstract}
We study fidelity decay in classically chaotic microwave billiards for a local, pistonlike boundary perturbation. We experimentally verify a predicted nonmonotonic crossover from the Fermi golden rule to the escape-rate regime of the Loschmidt echo decay with increasing local boundary perturbation. In particular, we observe pronounced oscillations of the decay rate as a function of the piston position which quantitatively agree with corresponding theoretical results based on a refined semiclassical approach for local boundary perturbations.
\end{abstract}

DOI: 10.1103/PhysRevE.83.016214

PACS number(s): 05.45.Mt, 03.65.Sq

\section{INTRODUCTION}

The stability of quantum time evolution measured by the overlap between time-evolved perturbed and unperturbed states, as suggested by Peres [1], has been studied from various viewpoints and under different names. In the field of quantum information this overlap is called "fidelity" [2] and plays an important role for quantifying the susceptibility of quantum dynamics to environmental or other external perturbations. In semiclassical quantum and wave mechanics, alternatively, the overlap of an initial state with the state reached after successive forward and backward time propagation, governed by the unperturbed and perturbed Hamiltonian, is often termed "Loschmidt echo" (LE) [3], especially for Hamiltonians associated with complex, in particular, classically chaotic dynamics. This terminology refers to the notion of echoes from momenta reversal in a Hamiltonian system considered by Loschmidt [4] in the 19th century.

For chaotic systems the LE has been predicted to exhibit different decay characteristics [5] depending on the form and strength of the perturbation. One distinguishes roughly three prominent LE decay regimes: the perturbative Gaussian [6,7], the Fermi-golden-rule (FGR) [5-8], and the Lyapunov regimes [5,9] (for reviews see Refs. [10,11]). The various perturbations considered have in common that they act "globally" on the system, i.e., already a moderate perturbation strength can cause a considerable rearrangement of the spectrum and eigenfunctions. Correspondingly, in a semiclassical picture, a global perturbation affects all trajectories of the system, and hence all of them are responsible for the decay of the LE. The corresponding, original semiclassical approach to the LE [5,9], which was recently generalized beyond the so-called diagonal approximation [12], was extended in Ref. [13] to strong local perturbations in coordinate space. This combined analytical and numerical study revealed for a billiard with a local boundary deformation, much larger than the de Broglie wavelength, a novel LE decay law $\exp (-2 \gamma t)$, where $\gamma$ is the classical "escape rate" from the related open billiard. This approach was refined and generalized to weak perturbations in Ref. [14] predicting a nonmonotonic crossover from the FGR to the escape-rate regime with increasing perturbation. For the case of a pistonlike boundary perturbation the LE decay rate is expected to show distinct oscillations as a function of the perturbation strength, i.e., piston position. While this nonmonotonic crossover has been numerically confirmed for maps [15], quantum wave packet simulations for billiards requiring more expensive numerics could only reveal precursors of this behavior [14], calling for an experimental verification of the oscillations.

For a global perturbation the fidelity decay was studied in a microwave billiard with classically chaotic dynamics by shifting a billiard wall [16]. Using the concept of scattering fidelity [17] the predicted fidelity decay from the perturbative to the FGR regime was verified experimentally.

A theoretical and experimental investigation of fidelity decay for another type of "local" perturbation in the perturbative regime, where the eigenstates are not significantly modified by the perturbation, has been done in [18]. On the experimental side a small scatterer was shifted inside the microwave billiard in a two-dimensional array of pointlike scatterers. Using the random plane wave conjecture, an algebraic decay $1 / t$ was predicted theoretically and confirmed experimentally. Another type of fidelity decay caused by local perturbations has been studied in Ref. [19], where the coupling to an attached antenna was varied. In the present paper we use a microwave billiard with a piston attached to address the predicted nonmonotonic features in the fidelity decay.

The paper is organized as follows. In Sec. II we present the experimental setup and introduce the scattering fidelity. In Sec. III we briefly summarize the semiclassical results for the LE decay with local boundary perturbations [14] and derive (in the Appendix) an extension of the expression for the effective decay rate for the case of a pistonlike boundary perturbation as used in the experiment. We then present in Sec. IV our results for the experimentally determined scattering fidelity decay and compare them with the theoretical predictions for the corresponding Loschmidt echo decay. Our main findings are then summarized in Sec. V.

\section{EXPERIMENT}

Microwave experiments with flat cavities have become a well-known paradigm in the field of quantum chaos [20]. In microwave billiards we can measure scattering matrix elements $S_{a b}(v)$ and $S_{a b}^{\prime}(v)$ for unperturbed and perturbed 


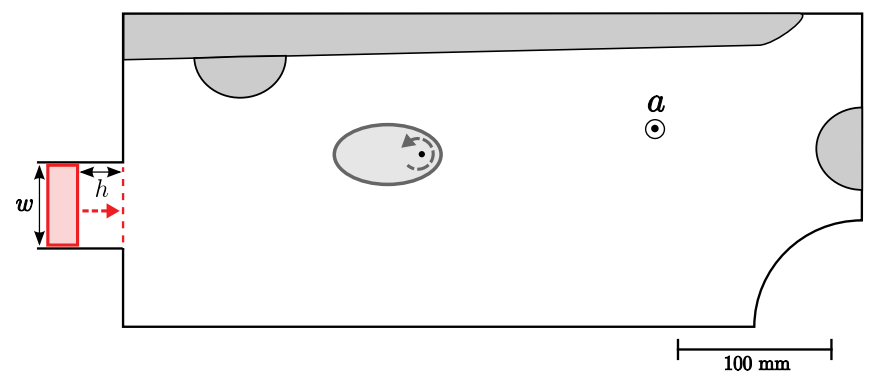

FIG. 1. (Color online) Geometry of the chaotic Sinai-shaped billiard (length of $472 \mathrm{~mm}$, width of $200 \mathrm{~mm}$, and a quartercircle of radius $70 \mathrm{~mm}$ ) with a variable pistonlike local boundary deformation. The piston position can be changed from a displacement $h=45 \mathrm{~mm}$ to $h=0 \mathrm{~mm}$ for four different piston widths $w=20,40,70$, and $98 \mathrm{~mm}$. At position $a$ the measuring antenna is introduced. The additional elements were inserted to perform ensemble averages (rotatable ellipse) and to reduce the influence of bouncing balls.

systems, independently, in frequency space. The scattering fidelity amplitude is defined in terms of their Fourier transforms, $\hat{S}_{a b}$ and $\hat{S}_{a b}^{\prime}$ (upon choosing an appropriate frequency window) [17]:

$$
f_{a b}(t)=\frac{\left\langle\hat{S}_{a b}(t) \hat{S}_{a b}^{\prime *}(t)\right\rangle}{\sqrt{\left\langle\hat{S}_{a b}(t) \hat{S}_{a b}^{*}(t)\right\rangle\left\langle\hat{S}_{a b}^{\prime}(t) \hat{S}_{a b}^{*}(t)\right\rangle}} .
$$

The scattering fidelity itself is

$$
F(t)=\left|f_{a b}(t)\right|^{2} .
$$

For chaotic systems and weak coupling of the measuring antenna the scattering fidelity approaches the ordinary fidelity [17].

In the present experiment we chose a resonator with a height of $8 \mathrm{~mm}$ which can be considered as two-dimensional for frequencies below $18 \mathrm{GHz}$. The setup, as illustrated in Fig. 1, is based on a quarter Sinai-shaped billiard. Additional elements were inserted into the billiard to reduce the influence of bouncing-ball resonances. The classical dynamics for the chosen geometry of the billiard is chaotic. The straight left boundary of the unperturbed billiard was deformed at a certain position by inserting pistons of four different widths $w$. The horizontal piston position can be changed in steps of $0.5 \mathrm{~mm}$ via a step motor from a displacement $h=45 \mathrm{~mm}$ to $h=0 \mathrm{~mm}$. At position $a$ an antenna is fixed and connected to an Agilent 8720ES vector network analyzer (VNA), which was used for measurements in a frequency range from 2 to $18 \mathrm{GHz}$ with a resolution of $0.1 \mathrm{MHz}$. We measured the reflection $S$-matrix element $S_{a a}$ for four piston widths and all displacements $h$ realizing 18 different positions of a rotating ellipse (see Fig. 1) to perform ensemble averages. The unperturbed system is defined as the one with the straight wall, corresponding to $h=0 \mathrm{~mm}$.

\section{THEORY}

The LE

$$
M(t)=\left|\left\langle\phi\left|e^{i H^{\prime} t / \hbar} e^{-i H t / \hbar}\right| \phi\right\rangle\right|^{2}
$$

is defined as the overlap of an initial state $|\phi\rangle$ evolved in time $t$ under a Hamiltonian $H$ with that evolved under a perturbed Hamiltonian $H^{\prime}$. Within a semiclassical approach this quantity was studied in Refs. [13,14] for local perturbations in chaotic systems. There it was shown that the LE is approximately [14]

$$
M(t) \approx e^{-\kappa \gamma t}
$$

with the effective decay rate $\kappa$ given by

$$
\kappa=2\left(1-\operatorname{Re}\left\langle e^{2 \pi i u / \lambda}\right\rangle\right) .
$$

Here $u$, called the deformation function, equals the length difference, induced by the local boundary perturbation, between the perturbed trajectory and the unperturbed one. $\lambda$ denotes the de Broglie wavelength. For the case of a pistonlike boundary deformation with piston width $w$ and displacement $h$ of the piston, as it is realized in our experiment, we find, in generalization of the results of Ref. [14] (see the Appendix),

$$
\begin{aligned}
\kappa=2 & -\frac{2}{w} \sum_{k=0}^{\infty} \int_{\Omega_{2 k+1}} d x d \theta \cos \theta \\
& \times \cos \left[\frac{4 \pi}{\lambda}(h \cos \theta+k w \sin \theta)\right]
\end{aligned}
$$

with the integration domains $\Omega_{2 k+1}$ over incident positions $x$ and momentum directions $\theta$ defined in Eq. (A4). In the limit $h \ll w$, Eq. (6) reduces to [14]

$$
\kappa=\pi \mathbf{H}_{1}(4 \pi h / \lambda)
$$

with $\mathbf{H}_{1}$ being the Struve $H$ function of first order.

Furthermore, $M(t)$ in Eq. (4) depends on $\gamma$, which is the classical escape rate of the corresponding open cavity if the piston is removed. It is given by

$$
\gamma=\frac{p_{0}}{m l_{d}}
$$

for particles with momentum $p_{0}$ and mass $m$, and for the average dwell length $l_{d}$ of paths in the related open chaotic billiard. In billiards with openings (deformation widths) $w$ much smaller than the perimeter one can approximate $l_{d} \approx$ $\pi A / w$ with $A$ the area of the corresponding closed billiard. Further, we will set $p_{0} / m=c$, where $c$ is, in the case of the microwave billiard, the speed of light.

In semiclassical theory of the Loschmidt echo the perturbation strength is a measure of the action change introduced by the perturbation. Thus, for perturbations caused by pistonlike boundary deformations the piston displacement $h$ serves as the measure of the perturbation strength. As shown in Ref. [14] it is convenient to define a dimensionless quantity $\chi=2 \pi \sqrt{\left\langle u^{2}\right\rangle} / \lambda$, where $\left\langle u^{2}\right\rangle=8 h^{2} / 3$ for a piston-type deformation, as the perturbation strength. Then, based on Eqs. (4) and (5), different decay regimes of the LE can be identified as follows. For weak local perturbations, $\chi \leqslant 1$, one has $M(t) \approx e^{-\chi^{2} \gamma t}$ characterizing the FGR regime. Strong local perturbations, $\chi \gg 1$, lead to $M(t) \approx e^{-2 \gamma t}$ corresponding to the escape-rate regime. In the following section we will use Eq. (4), together with the refined expression (6) for the decay rate $\kappa$, for a comparison with the experimentally determined scattering fidelity (2). 


\section{RESULTS AND DISCUSSION}

In this section we present our measurements of the scattering fidelity decay for the pistonlike boundary perturbation and compare them with the theoretical predictions (4)-(7) for LE decay for this specific type of perturbation. We start with a piston of width $w=40 \mathrm{~mm}$. In Fig. 2 the scattering fidelity $F(t)$, Eq. (2), is plotted for three different piston displacements $h$ acting as perturbation to the system (symbols and solid lines). Additionally, the corresponding semiclassical predictions for the Loschmidt decay according to Eq. (4) are plotted (dashed lines) with $\kappa$ used as a fitting parameter, while $\gamma$ was obtained from the geometry. The experimental fidelity decay shows good agreement with the expected exponential law beyond a certain time, which passes until the perturbation is "seen" during the measuring process. Upon increasing the displacements $h$, illustrated in Fig. 2 by the successive triangle (blue), circle (green), and squares (red) traces, the corresponding LE decay exponent $\kappa$ exhibits a nonmonotonic behavior.

For a more detailed investigation of this dependence of the Loschmidt decay exponent $\kappa$ on the displacements $h$ of the piston, $\kappa$ is compared to the corresponding theoretical predictions in Fig. 3. The data points $\kappa(h)$, obtained from fitting to the experimental fidelity decay results as in Fig. 2, are shown by asterisks. The three cases discussed in Fig. 2 are marked by correspondingly colored symbols at $h_{1}=1,5$, and $10 \mathrm{~mm}$. The figure shows an oscillating behavior of the fidelity exponent, and thereby of the fidelity decay at fixed time, as already predicted and referred to as Fabry-Perot-type interferences between perturbed and unperturbed paths in Ref. [14]. In Fig. 3 the semiclassical results for $\kappa(h)$ resulting from the numerical evaluation of the expression (6) and the approximation (7) (for $h \ll w$ ) are depicted as solid and dashed curves, respectively. Already the dashed curve exhibits qualitatively good agreement with the measurement,

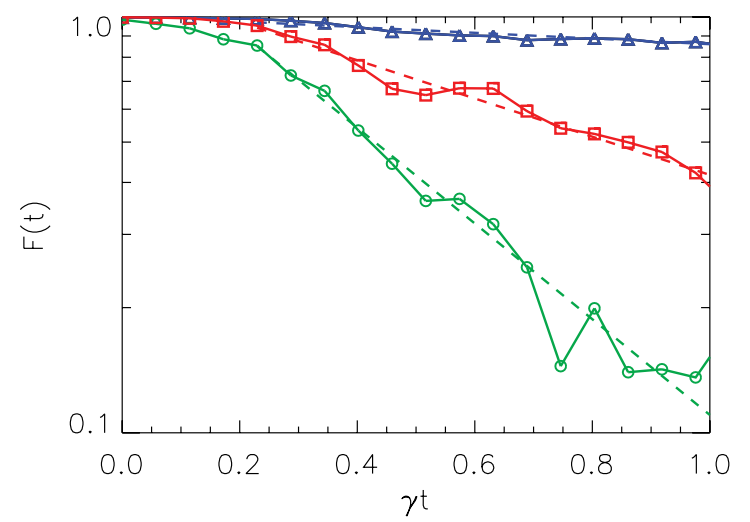

FIG. 2. (Color online) Measured scattering fidelity decay $F(t)$, Eq. (2) (solid lines with symbols) for three different piston displacements $h_{1}=1 \mathrm{~mm}$ (blue triangles), $h_{2}=5 \mathrm{~mm}$ (green circles), $h_{3}=$ $10 \mathrm{~mm}$ (red squares), for a frequency range $17-18 \mathrm{GHz}$ corresponding to a mean de Broglie wavelength $\bar{\lambda} \approx 17 \mathrm{~mm}$. The dashed lines show the corresponding semiclassical prediction, Eq. (4), for the LE decay, with $\kappa$ chosen as free parameter: $\kappa_{1}=0.26, \kappa_{2}=2.78$, and $\kappa_{3}=1.09$, respectively. The time is given in units of the dwell time $1 / \gamma$, with $\gamma$ determined from experimental parameters via Eq. (8) with $w=40 \mathrm{~mm}$.

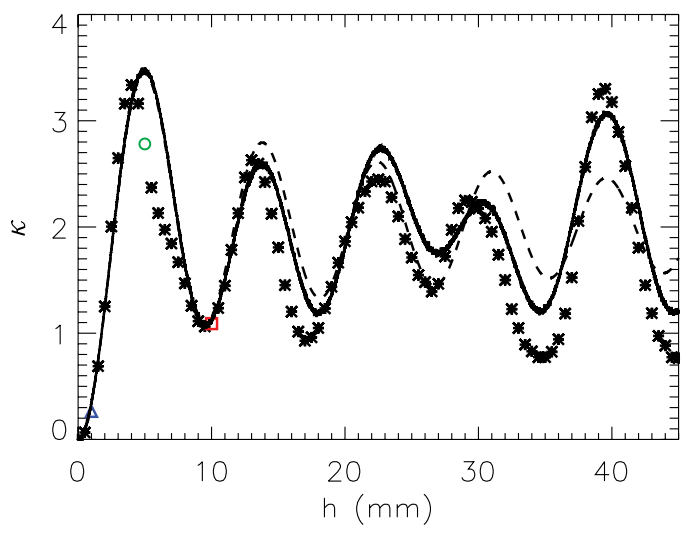

FIG. 3. (Color online) Decay rate $\kappa$ as a function of piston displacement $h$ for a piston of width $w=40 \mathrm{~mm}$ in a frequency range $17-18 \mathrm{GHz}$ corresponding to a mean de Broglie wavelength $\bar{\lambda} \approx 17 \mathrm{~mm}$. The asterisks represent the data points obtained from fitting the decay exponent of the measured scattering fidelity. The three cases discussed in Fig. 2 are marked by correspondingly colored symbols. The dashed curve shows the theoretical approximation (7) (valid for $h \ll w$ ), and the solid curve is a result of the numerical evaluation of the full semiclassical expression (6).

though there is a mismatch in the amplitudes: While this approximative theoretical result shows a monotonic decay of the maximum amplitude with $h$, the experimentally observed peaks of $\kappa(h)$ do not show this simple structure. However, the refined semiclassical prediction (6) (solid line) reflects the experimentally found irregular oscillation of amplitudes much more convincingly, showing reasonable agreement. In particular, for $h \approx w=40 \mathrm{~mm}$ (square shape of the pistonlike deformation) the experimental results show a particularly pronounced amplitude which is met by the solid line. In this range, which is beyond the range of validity of Eq. (7), the expression (6) constitutes a clear improvement.

Furthermore, in Fig. 4 we present on the same plot experimental data for $\kappa$ versus $\chi$ curves with the mean de Broglie wavelength $\bar{\lambda}$ in the frequency range $\bar{\lambda}<2 w$, while $w \geqslant 40 \mathrm{~mm}$ and $h<w$. We find very convincing agreement with the theoretical prediction (7).

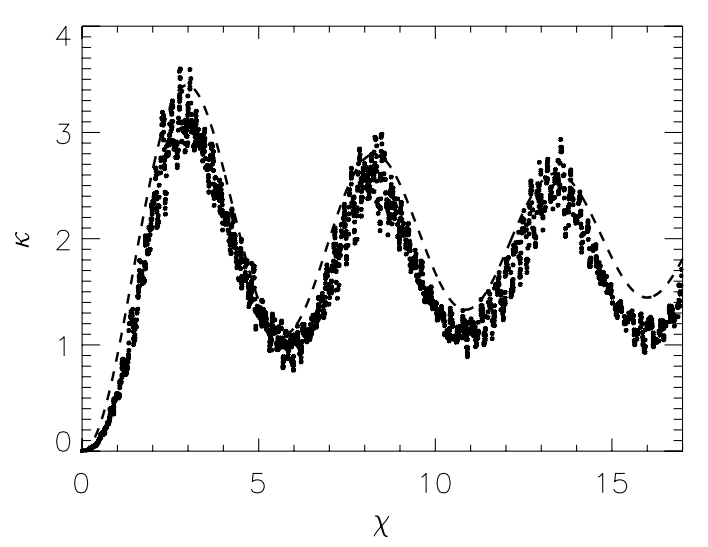

FIG. 4. Decay rate $\kappa$ as a function of $\chi=\sqrt{8 / 3} h 2 \pi / \bar{\lambda}$. The fuzzy trace depicts the overlayed experimental data and the dashed curve the theoretical prediction (7). 


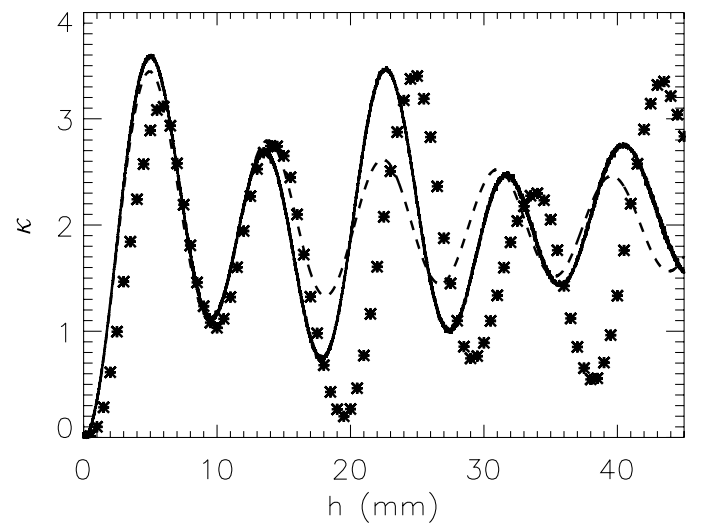

FIG. 5. Decay rate $\kappa$ as a function of the displacement $h$ for a thin piston of width $w=20 \mathrm{~mm}$ in a frequency range $17-18 \mathrm{GHz}$ corresponding to a mean de Broglie wavelength $\bar{\lambda} \approx 17 \mathrm{~mm}$. The asterisks show the data points extracted again from the fit exponent of the exponential decay of the observed scattering fidelity. The dashed and solid curves show the theoretical predictions based on Eq. (7) and the numerical evaluation of Eq. (6).

Finally, we demonstrate that the agreement between the experimental and theoretical curves can be shaken by pushing the experimental conditions too far beyond the main limit of the semiclassical theory, $\lambda \ll w$. Figure 5 shows the decay rate $\kappa$ for a piston width $w=20 \mathrm{~mm}$ which is of the order of $\bar{\lambda}$. As expected, the agreement between theory and experiment is not as good as that for the $w=40 \mathrm{~mm}$ case; in particular, the experimental data points (dashed line) oscillate with a period that differs from the theoretical one. Experimentally, we again find a more pronounced amplitude around $h \approx w$, which is again described more convincingly by the full (solid line) than the approximative theoretical expression. However, the fact that the experimental parameters are beyond the regime of validity of the semiclassical theory does not allow for a further reasonable comparison between experiment and theory.

\section{CONCLUSIONS}

In this work we presented an experimental verification of the recent semiclassical predictions for fidelity decay arising from a local perturbation of a chaotic quantum system. In particular, we could confirm that the rate governing exponential fidelity decay exhibits oscillations as a function of the perturbation strength. The observed nonmonotonic behavior implies that for certain ranges of the perturbation strength the fidelity decay becomes weaker (for fixed time) with increasing perturbation strength. While the original semiclassical treatment [14] for a piston-type local boundary deformation was based on the assumption of a small piston displacement, the present microwave setting required a generalization of the semiclassical approach to arbitrary ratios between piston displacement and width, which we performed by deriving an expression for the decay exponent in terms of a quadrature. We find quantitative agreement between the measurements and this refined semiclassical theory despite the fact that the microwave billiard does not really satisfy the underlying semiclassical assumption, namely, that the extent of the local perturbation, here the piston width $w$, should be much larger than the de Broglie wavelength $\lambda$. An improved semiclassical approach for local perturbations of size $w \lesssim \lambda$ would require one to treat semiclassical contributions due to diffractive trajectories properly, which is left for future research.

On the experimental side, there remains the challenge to observe fidelity decay in the escape-rate regime (for strong perturbations) characterized by a perturbation-independent fidelity decay rate. Naturally, this regime is difficult to access since the expected signals are tiny.

\section{ACKNOWLEDGMENTS}

We thank Thomas Seligman for helpful discussions at an early stage, which partly triggered this work. We are also thankful to Rodolfo Jalabert for helpful correspondence. B.K., U.K., H.-J.S., and K.R. acknowledge funding from the Deutsche Forschungsgemeinschaft through the research group FOR760 "Scattering Systems with Complex Dynamics," A.G. acknowledges the support by EPSRC under Grant No. EP/E024629/1.

\section{APPENDIX: SEMICLASSICAL THEORY OF EXPONENTIAL FIDELITY DECAY FOR ARBITRARY $w$ and $h$}

In view of the above experiments we extend the semiclassical theory for the decay of the fidelity due to pistonlike boundary deformations, presented in Ref. [14] for the limit $h \ll w$. There it was shown that the rate of exponential fidelity decay is given by Eq. (5) with

$$
\left\langle e^{2 \pi i u / \lambda}\right\rangle=\int_{0}^{w} \frac{d x}{w} \int_{0}^{\pi / 2} d \theta \cos \theta e^{2 \pi i u(x, \theta) / \lambda} .
$$

Here $x$ and $\theta$ denote the incident position and angle, respectively [see Fig. 6(a)]. The deformation function $u(x, \theta)$ equals the length difference between the perturbed and unperturbed trajectory of a correlated trajectory pair. A pair made up of an unperturbed and perturbed trajectory is considered correlated if the two trajectories exit the perturbation region with the same momentum direction [14] (see Fig. 6).

We first note that only perturbed trajectories with an odd number of reflections may exit the perturbation region with

(a)

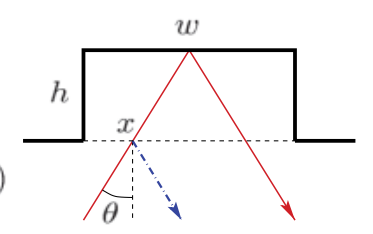

(b)
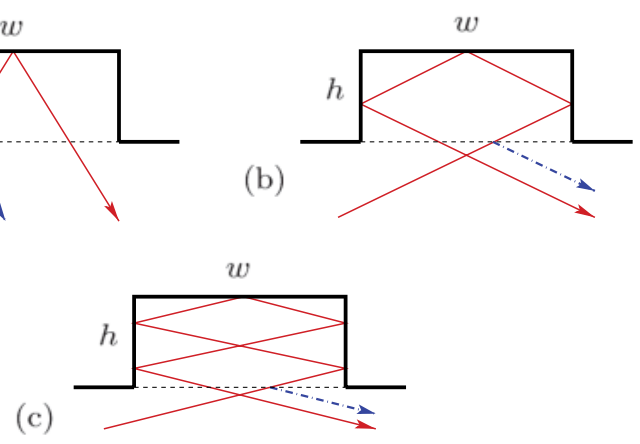

FIG. 6. (Color online) Examples of correlated trajectory pairs, unperturbed (blue dash-dotted line) and perturbed (red solid line), belonging to sets $\Omega_{1}$ (a), $\Omega_{3}$ (b), and $\Omega_{5}$ (c) (see text in the Appendix). 
(a)

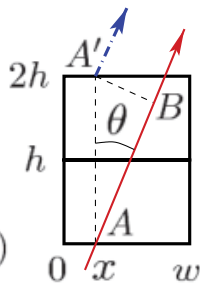

(b)

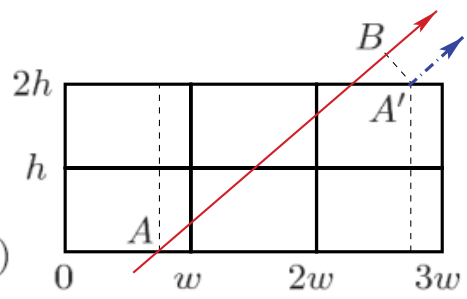

(c)

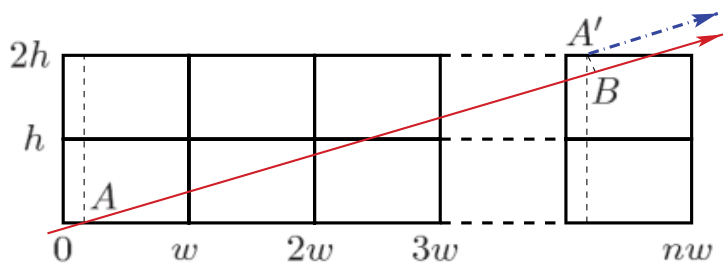

FIG. 7. (Color online) "Unfolded" representation of correlated trajectory pairs belonging to sets $\Omega_{1}$ (a), $\Omega_{3}$ (b), and $\Omega_{n}$ (c) with an odd integer $n$.

the same momentum direction as the unperturbed trajectory and, therefore, contribute to the fidelity. We denote by $\Omega_{n}$ a set of all correlated trajectory pairs where the perturbed trajectory exhibits $n$ reflections. The panels (a)-(c) in Fig. 6 show representative trajectory pairs belonging to the sets $\Omega_{1}, \Omega_{3}$, and $\Omega_{5}$, respectively. Equation (A1) can then be written as

$$
\left\langle e^{2 \pi i u / \lambda}\right\rangle=\frac{1}{w} \sum_{k=0}^{\infty} \int_{\Omega_{2 k+1}} d x d \theta \cos \theta e^{i u / \lambda},
$$

where the double integral in the $k$ th summand runs over a region in the $(x, \theta)$ plane that defines the set $\Omega_{2 k+1}$.

In order to calculate the deformation function $u(x, \theta)$ for a trajectory pair from the set $\Omega_{n}$ we "unfold" the perturbation rectangle (by "gluing" mirror copies of the rectangle along the reflection sides) making the perturbed trajectory become a straight line (see Fig. 7). Thereby, Fig. 7(a) is the "unfolded" version of Fig. 6(a), Fig. 7(b) corresponds to Fig. 6(b), and Fig. 7(c) represents a trajectory pair belonging to $\Omega_{n}$. In this representation, the deformation function $u$ equals the length of the interval $A B, u=\overline{A B}$. Here $A$ is a point of incidence, while the point $B$ belongs to the perturbed trajectory and is specified by requiring the angle $\widehat{A B A^{\prime}}$ to be $\pi / 2$, where $A^{\prime}$ represents

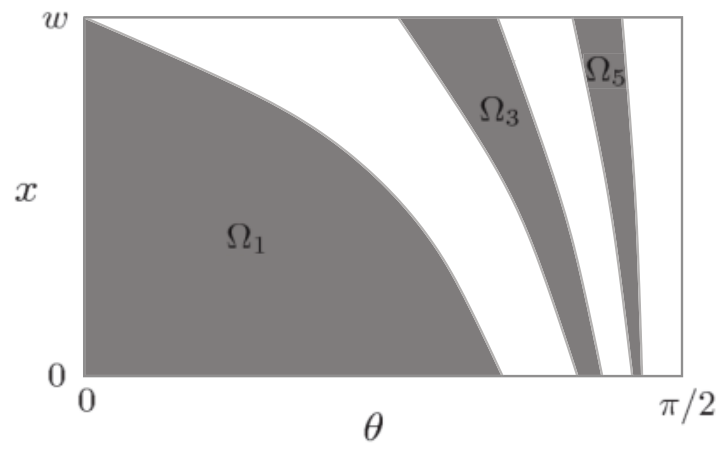

FIG. 8. Schematic representation of the regions $\Omega_{1}, \Omega_{3}$, and $\Omega_{5}$ [see Eq. (A4)]. Further regions, $\Omega_{2 k+1}$ with $k \geqslant 3$, contributing to the sum on the right-hand side of Eq. (6) are not shown in the figure; they cluster as narrow stripes "to the right" of $\Omega_{5}$ and approach $\theta=\pi / 2$ in the limit $k \rightarrow \infty$.

the incident point $A$ in the "exit" copy of the perturbation rectangle (see Fig. 7). Then, a geometrical calculation yields

$$
u(x, \theta)=2 h \cos \theta+(n-1) w \sin \theta,
$$

where $(x, \theta) \in \Omega_{n}$.

We now give a precise definition of the region $\Omega_{n}$ in the $(x, \theta)$ plane. As evident from Fig. 7(c), a trajectory pair belongs to $\Omega_{n}$ if and only if the $x$ coordinate of the exit point of the perturbed trajectory (in the unfolded picture) lies between $(n-1) w$ and $n w$. This yields

$$
\begin{aligned}
\Omega_{n}= & \{(x, \theta): x \in(0, w), \quad \theta \in(0, \pi / 2), \\
& x+2 h \tan \theta \in(n-1, n) w\} .
\end{aligned}
$$

Figure 8 schematically shows the first three sets $\Omega_{1}, \Omega_{3}$, and $\Omega_{5}$, contributing to the sum in Eq. (A2).

Combining Eqs. (5), (A2), (A3), and (A4) we arrive at Eq. (6), which completes the derivation. The sum and integrals in Eq. (6) are then computed numerically by means of Monte Carlo sampling.

As a final remark, we note that in the limit of $h \ll w$ the sum on the right-hand side of Eq. (6) is dominated by the $k=$ 0 term. Then the integration region $\Omega_{1}$ can be approximately extended to the rectangle $x \in(0, w), \theta \in(0, \pi / 2)$, since the contribution for large angles, $\theta$ close to $\pi / 2$, is suppressed by the $\cos \theta$ term in the integrand. This approximation leads to Eq. (7) (see also Ref. [14]).
[1] A. Peres, Phys. Rev. A 30, 1610 (1984).

[2] M. A. Nielsen and I. L. Chuang, Quantum Computation and Quantum Information (Cambridge University Press, Cambridge, 2000).

[3] G. Usaj, H. M. Pastawski, and P. R. Levstein, Mol. Phys. 95, 1229 (1998).

[4] J. Loschmidt, Sitzungsber. Akad. Wiss., Wien, II 73, 128 (1876).

[5] R. A. Jalabert and H. M. Pastawski, Phys. Rev. Lett. 86, 2490 (2001).

[6] P. Jacquod, P. G. Silvestrov, and C. W. J. Beenakker, Phys. Rev. E 64, 055203(R) (2001).
[7] N. R. Cerruti and S. Tomsovic, Phys. Rev. Lett. 88, 054103 (2002).

[8] T. Prosen, Phys. Rev. E 65, 036208 (2002).

[9] F. M. Cucchietti, C. H. Lewenkopf, E. R. Mucciolo, H. M. Pastawski, and R. O. Vallejos, Phys. Rev. E 65, 046209 (2002).

[10] T. Gorin, T. Prosen, T. H. Seligman, and M. Žnidarič, Phys. Rep. 435, 33 (2006).

[11] P. Jacquod and C. Petitjean, Adv. Phys. 58, 67 (2009).

[12] B. Gutkin, D. Waltner, M. Gutiérrez, J. Kuipers, and K. Richter, Phys. Rev. E 81, 036222 (2010).

[13] A. Goussev and K. Richter, Phys. Rev. E 75, 015201(R) (2007). 
[14] A. Goussev, D. Waltner, K. Richter, and R. A. Jalabert, New J. Phys. 10, 093010 (2008).

[15] N. Ares and D. A. Wisniacki, Phys. Rev. E 80, 046216 (2009).

[16] R. Schäfer, H.-J. Stöckmann, T. Gorin, and T. H. Seligman, Phys. Rev. Lett. 95, 184102(2005).

[17] R. Schäfer, T. Gorin, T. H. Seligman, and H.-J. Stöckmann, New J. Phys. 7, 152 (2005).
[18] R. Höhmann, U. Kuhl, and H.-J. Stöckmann, Phys. Rev. Lett. 100, 124101 (2008).

[19] B. Köber, U. Kuhl, H.-J. Stöckmann, T. Gorin, D. V. Savin, and T. H. Seligman, Phys. Rev. E 82, 036207 (2010).

[20] H.-J. Stöckmann, Quantum Chaos-An Introduction (Cambridge University Press, Cambridge, 1999). 